\title{
Avaliação das alterações na distância intercaninos do arco inferior em pacientes com má oclusão de Classe I tratados ortodonticamente*
}

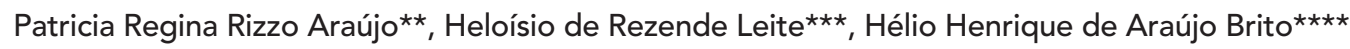

Resumo

Introdução: modificações na distância intercaninos do arco inferior, durante o tratamento ortodôntico, são consideradas, por alguns pesquisadores, como instáveis. Objetivo e Metodologia: o presente estudo, com o propósito de observar possiveis alterações pós-tratamento na distância intercaninos, decorrentes da expansão do arco nessa região, avaliou 30 pacientes com má oclusão inicial de Classe I de Angle, tratados ortodonticamente no COP (Centro de Odontologia e Pesquisa) da PUC (Pontifícia Universidade Católica) de Minas Gerais. A distância intercaninos inferior foi medida de duas maneiras: na ponta da cúspide e na face vestibular dos caninos. As medidas foram feitas em três tempos: T1- pré-tratamento, T2- pós-tratamento e T3- após um mínimo de um ano sem uso da contenção inferior. Foram realizadas comparações quanto ao gênero, realização ou não de exodontias no tratamento, tempo fora de contenção (de um a cinco anos e acima de cinco anos) e tipo de movimentação dos caninos (translação ou inclinação). Resultados e Conclusões: houve um aumento significantes da distância intercaninos durante o tratamento ortodôntico e um decréscimo no período pós-contenção, tanto na ponta da cúspide quanto na face vestibular. Entretanto, a redução da medida, no período pós-contenção, foi maior na ponta da cúspide do que na face vestibular. Não foram observadas diferenças estatisticamente significantes na recidiva, com relação ao gênero e ao tempo fora de contenção. Pôde-se observar, porém, uma maior recidiva da distância intercaninos (referência na ponta da cúspide) nos casos tratados com extração de pré-molares. Entretanto, ao avaliar os valores obtidos na face vestibular, observou-se que, nesse grupo de pacientes, a expansão foi significantemente maior. Nos casos em que a expansão da distância intercaninos foi realizada através de translação do canino (50\% da amostra), houve uma maior recidiva na medida feita na ponta da cúspide do que na da face vestibular após a remoção das contenções, indicando que houve uma inclinação dos caninos para lingual.

Palavras-chave: Distância intercaninos. Expansão. Recidiva.

* Baseado em monografia apresentada ao Centro de Odontologia e Pesquisa da PUC-Minas, como requisito parcial à obtenção do título de especialista em Ortodontia e Ortopedia Facial.

** Especialista em Ortodontia e Ortopedia Facial pela PUC-Minas.

*** Mestre em Ortodontia pela Universidade de Pittsburgh (U.S.A.). Professor de Ortodontia da PUC-Minas.

**** Mestre em Ortodontia pela Universidade Federal do Rio de Janeiro. Professor de Ortodontia da PUC- Minas. 


\section{REVISÃO DE LITERATURA}

A distância intercaninos, ou distância bicaninos, é a medida da largura do arco dentário na região de caninos. Essa dimensão pode ser medida na ponta da cúspide ou no centro da faceta de desgaste, do canino de um lado do arco ao canino contralatera1 ${ }^{17,24,40}$. Outros pontos de referência utilizados para esta medição são a margem gengival distal ${ }^{39}$, a região cervical da face lingual ${ }^{29}$, a região mais cervical da face vestibular ${ }^{32}$ ou, ainda, a porção mais proeminente dessa mesma face dos caninos ${ }^{52}$.

A expansão da distância intercaninos no tratamento ortodôntico, como método de obtenção de espaço para o alinhamento dos dentes no arco inferior, vem sendo amplamente discutida. O conceito, difundido por Angle², em 1907, de que a oclusão ideal deveria conter todos os dentes, levou muitos profissionais a realizarem seus tratamentos através da expansão dos arcos. Acreditava-se que esse seria o único caminho para assegurar a estabilidade do tratamento. Para Angle ${ }^{2}$, a recidiva no apinhamento dentário, após a expansão dos arcos, significava que uma oclusão ideal não tinha sido alcançada.

A controvérsia entre tratamento com ou sem expansão se estende até os dias atuais. Strang ${ }^{47}$, em 1949, já ressaltava a importância da distância intercaninos original do arco mandibular como guia infalível para a manutenção do equilíbrio muscular. Rossouw e Preston ${ }^{37}$ reafirmaram a importância da manutenção da distância intercaninos durante o tratamento ortodôntico e declararam que os caninos representam as pedras fundamentais ou os alicerces dos arcos dentários.

Entretanto, a expansão da distância intercaninos inferior não pode ser desprezada como um meio de se conseguir aliviar o apinhamento dentário. Mesmo casos que incluem a extração de dentes podem apresentar alguma expansão lateral ao final do tratamento. Ricketts et al. ${ }^{34}$ desenvolveram um guia para previsão do aumento no perímetro do arco, associado à expansão ortodôntica. Segundo esses autores, para cada milímetro de expansão da distância intercaninos, ocorre um milímetro de aumento no perímetro do arco. Germane et al. ${ }^{18}$ desenvolveram, através de um programa de computador, uma forma de arco para quantificar os aumentos no perímetro produzidos pela expansão. Esses autores observaram que o primeiro milímetro de expansão dos caninos resultou em $0,73 \mathrm{~mm}$ de ganho no perímetro. A cada milímetro a mais de expansão, o ganho em perímetro foi maior. $\mathrm{O}$ ganho total no perímetro, resultante de $5 \mathrm{~mm}$ de expansão da distância intercaninos, chegou a $5,34 \mathrm{~mm}$.

A maior polêmica em relação à expansão dos caninos inferiores diz respeito à estabilidade da alteração conseguida após o tratamento. Diversos autores $^{1,8,11,14,15,19,23,25,26,27,28,30,33,46,49}$ relataram que todo aumento da distância intercaninos durante o tratamento ortodôntico seria seguido de uma constrição no período pós-contenção. Entretanto, alguns autores $21,35,36,40,41,51$ defendem que alguma expansão do arco inferior pode ser mantida após o tratamento ortodôntico. Outros autores ${ }^{5,6,11}$ afirmam, ainda, que parte dessa redução após o período de contenção pode estar associada ao desenvolvimento normal dos arcos dentários.

As alterações que ocorrem durante o desenvolvimento normal dos arcos dentários influenciam também aqueles tratados ortodonticamente ${ }^{31}$. Segundo Horowitz e Hixon ${ }^{22}$, o tratamento ortodôntico pode alterar temporariamente o curso das mudanças fisiológicas. Entretanto, após o período de intervenção, o processo de maturação tem continuidade.

De acordo com a literatura revista, existe uma variação da distância intercaninos com a idade.

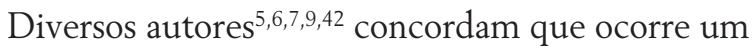
aumento desta dimensão com a erupção dos incisivos inferiores e uma redução na idade adulta. Apenas Harris ${ }^{20}$ encontrou um aumento da distância intercaninos inferior na idade adulta, porém sem significância estatística.

Strang ${ }^{48}$ acreditava que, em casos de extração dos pré-molares inferiores, seria permissível que 
ocorresse uma expansão vestibular dos caninos, pois esses dentes estariam sendo movidos para uma região mais ampla do arco e o equilíbrio muscular não seria violado.

Entretanto, outros autores ${ }^{4,12,23,24,46}$ observaram que não existe diferença no comportamento dessa dimensão entre os pacientes tratados com e sem exodontia.

Uma amostra de 72 pacientes, 50 com má oclusão Classe I e 22 com má oclusão Classe II, tratados ortodonticamente, foi reexaminada alguns anos após a remoção das contenções (de 12 a 35 anos) por Davis e BeGole ${ }^{12}$. Vinte e seis casos haviam sido tratados com extração de quatro prémolares e quarenta e seis sem extração. Os autores explicam que a maior quantidade de expansão dos caninos, nos casos tratados com extração de prémolares, ocorre com a sua distalização para uma área mais larga do arco. Houve uma redução da distância intercaninos, estatisticamente significante, em ambos os grupos, no período pós-contenção, sem que houvesse diferença entre os grupos.

Segundo Fastlicht ${ }^{16}$, arcos mais estreitos, associados a uma extrusão dos caninos superiores e inferiores e a movimentos laterais da mandíbula, durante a mastigação, produzem um deslocamento leve, mas constante, dos caninos (os superiores labialmente e os inferiores lingualmente).

Shapiro ${ }^{40}$ relata uma diferença na recidiva da distância intercaninos inferior nos diferentes tipos de má oclusão. Segundo esse autor, os pacientes

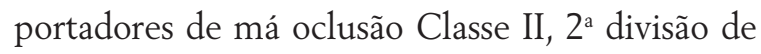
Angle, têm maior capacidade de manter a expansão conseguida durante o tratamento. Little, Wallen e Riedel ${ }^{24}$ não encontraram diferença significante entre as várias classes de má oclusão de Angle e a estabilidade, em longo prazo, da distância intercaninos. Burke et al. ${ }^{10}$, comparando o trabalho de diversos autores, concluíram que nenhum tipo de má oclusão pode ser associado a expansões bem sucedidas da distância intercaninos.

A estabilidade da expansão conseguida também pode ser afetada por fatores como o componente anterior da força oclusal ${ }^{44,45}$, alterações no equilíbrio entre a musculatura peribucal e a língua $32,35,46,48,50$, existência de hábitos anormais ${ }^{35,48}$, período de utilização da contenção inferior ${ }^{21,38}$, existência de tratamento interceptador com expansão do arco inferior ${ }^{13,26}$ ou recidiva do apinhamento ântero-inferior ${ }^{16,24,49,53}$.

Muitos foram os trabalhos que avaliaram as alterações pós-tratamento da distância intercaninos inferior, inclusive comparando as diferenças na recidiva entre os gêneros e entre os casos tratados com extração e sem extração de pré-molares ${ }^{4,12,23,24,37,44}$. Porém, em nenhum deles foram feitas comparações com relação ao tipo de movimento realizado na expansão dos caninos (translação ou inclinação) e à quantidade de recidiva observada.

O presente trabalho teve como propósitos: a) avaliar as alterações na distância intercaninos do arco inferior, promovidas com o tratamento ortodôntico, e seu comportamento após a remoção do aparelho de contenção; b) observar se existem diferenças nas alterações pós-tratamento relacionadas ao gênero, à realização de extrações no tratamento e ao tempo sem uso de contenções; c) comparar as alterações pós-contenção da distância intercaninos de tratamentos com expansão obtida através de translação dos caninos e de inclinação vestibular desses dentes.

\section{MATERIAL E MÉTODOS}

\section{Amostra}

Foram avaliados 30 pacientes, 23 do gênero feminino e 7 do gênero masculino, com má oclusão Classe I de Angle, submetidos a tratamento ortodôntico no Centro de Odontologia e Pesquisa da Pontifícia Universidade Católica de Minas Gerais. O tratamento foi realizado com extração em 12 pacientes e sem extração em 18; 25 pacientes estavam sem contenção entre um e cinco anos e 5 pacientes há mais de cinco anos. A idade média dos pacientes foi de 14 anos, com desvio-padrão de 2 anos e 7 meses (Tab. 1, 2). 
Os critérios para a seleção dos pacientes foram:

a) má oclusão Classe I de Angle antes do tratamento;

b) registros de diagnóstico completos nas fases de pré e pós-tratamento;

c) caninos permanentes inferiores irrompidos na fase pré-tratamento;

d) dentadura permanente completa, irrompida ou não, no início do tratamento, ou seja, sem

Tabela 1 - Caracterização da amostra.

\begin{tabular}{ccc}
\hline \multirow{2}{*}{ característica } & \multicolumn{2}{c}{ frequiência } \\
\cline { 2 - 3 } & $\mathbf{n}$ & $\%$ \\
\hline faixa etária & 9 & 30 \\
\hline até 12 anos & 7 & 23,3 \\
de 12 a 14 anos & 10 & 33,3 \\
de 14 a 16 anos & 4 & 13,4 \\
acima de 16 anos & 30 & 100 \\
total & & \\
\hline gênero & 23 & 76,7 \\
\hline feminino & 7 & 23,3 \\
masculino & 30 & 100,0 \\
total & & \\
\hline extração de pré-molares & 12 & 40,0 \\
\hline sim & 18 & 60,0 \\
não & 30 & 100,0 \\
total & & \\
\hline tempo sem contenção & 25 & 83,3 \\
\hline de 1 a 5 anos & 5 & 16,7 \\
acima de 5 anos & 30 & 100,0 \\
\hline total & &
\end{tabular}

Tabela 2 - Caracterização dos pacientes quanto à idade cronológica em T1, T2 e T3.

\begin{tabular}{ccccc}
\hline \multirow{2}{*}{ avaliação } & \multicolumn{4}{c}{ medidas descritivas } \\
\cline { 2 - 5 } & mínimo & máximo & média & desvio-padrão \\
\hline T1 & 10 a $9 \mathrm{~m}$ & $20 \mathrm{a} 7 \mathrm{~m}$ & $14 \mathrm{a}$ & $2 \mathrm{a} 7 \mathrm{~m}$ \\
$\mathrm{~T} 2$ & $13 \mathrm{a} 6 \mathrm{~m}$ & $23 \mathrm{a} 8 \mathrm{~m}$ & $16 \mathrm{a} 6 \mathrm{~m}$ & $2 \mathrm{a} 7 \mathrm{~m}$ \\
T3 & $16 \mathrm{a} 9 \mathrm{~m}$ & $29 \mathrm{a} 8 \mathrm{~m}$ & $21 \mathrm{a} 6 \mathrm{~m}$ & $3 \mathrm{a} 1 \mathrm{~m}$ \\
\hline
\end{tabular}

Nota: T1= antes do tratamento;

T2= após o tratamento;

T3= pelo menos um ano após a retirada da contenção. agenesias;

e) sem perdas dentárias após o fim do tratamento ortodôntico (no período de contenção ou pós-contenção);

f) mínimo de um ano sem uso da contenção inferior (fixa ou removível);

g) ausência de síndromes e/ou alterações sistêmicas;

h) ausência de hábitos anormais de sucção;

i) ausência de periodontopatias.

\section{Método de registro}

Foram utilizados 90 modelos do arco inferior em três épocas: T1 - pré-tratamento; T2 - pós-tratamento e T3 - após um período sem uso de contenção inferior, que variou de um a oito anos, nos pacientes estudados.

Os dados do paciente foram registrados em uma ficha clínica, que continha seus dados pessoais, e um questionário sobre o período de contenção e pós-contenção. Esses dados foram utilizados para a conferência com os dados presentes no arquivo do paciente.

As anotações referentes às medidas realizadas nos modelos de estudo foram realizadas em uma ficha desenvolvida para essa finalidade.

\section{Método de medida}

Foi utilizado um paquímetro quadrimensional (Digimess, São Paulo/SP), com 0,05mm de resolução, para medir a distância do canino permanente de um lado ao canino permanente do outro lado do arco inferior (Fig. 1A). Foi utilizada, como referência, a ponta da cúspide dessa unidade dentária (Fig. 1B). Alguns casos apresentaram desgaste no topo das cúspides dos caninos. Nesses pacientes, as medidas foram feitas de acordo com a sugestão de Barrow e White ${ }^{3}$, em um ponto de aproximação do local original da ponta da cúspide sobre o centro da faceta de desgaste. O paquímetro foi mantido paralelo ao plano oclusal inferior, para que a medida fosse realizada unicamente no sentido horizontal. 
Foram realizadas medidas da distância intercaninos utilizando-se também a face vestibular dos caninos, num ponto mais cervical da coroa, no longo eixo do dente (Fig. 1C). A comparação das alterações nas duas dimensões medidas informa quanto ao tipo de movimento: translação ou inclinação. Se a extensão do movimento na ponta da cúspide for maior que na porção cervical do dente, conclui-se que houve uma inclinação do mesmo para vestibular. Porém, se a quantidade de movimento em ambos os locais utilizados como referência (ponta da cúspide e porção cervical da face vestibular) for igual, o movimento conseguido é de translação.

\section{Análise estatística}

Com o objetivo de avaliar as mudanças da distância intercaninos durante o tratamento (T2-T1), no período pós-contenção (T3-T2) e no período total do estudo (T3-T1), utilizou-se o teste de Friedman. Trata-se de um teste não paramétrico para amostras dependentes.

A amostra foi, em seguida, separada em subgrupos, de acordo com o gênero, a existência ou não de extrações no tratamento e o tempo sem uso de contenção inferior. Para avaliar o efeito desses fatores nas alterações da distância intercaninos, utilizou-se o teste de Kruskal-Wallis, que é um teste não paramétrico para amostras independentes. Foi observado, também, o tipo de movimento realizado nos caninos, comparando-se a quantidade de movimento da ponta da cúspide com a quanti- dade de movimento da porção cervical do dente. A analise deste item foi feita através do teste exato de Fischer.

Utilizou-se o teste do Qui-quadrado para avaliar a influência do gênero, da existência de extração e do tempo sem contenção na categorização dos indivíduos em grupos, conforme o tipo de movimento realizado nos caninos (translação ou inclinação). Em alguns casos, utilizou-se o teste exato de Fisher, que tem o mesmo objetivo do teste Qui-quadrado.

Para avaliar o erro de medição, foram selecionados, ao acaso, 30 modelos de 10 pacientes da amostra e as medidas foram refeitas por outro examinador (Gráf. 1). A comparação entre os dois avaliadores foi realizada utilizando-se o teste $t$ de Student para amostras pareadas, que tem como objetivo avaliar a equivalência entre duas medidas realizadas em um mesmo indivíduo.

Foi adotado, para todos os testes, o nível de significância de $5 \%$.

\section{RESULTADOS}

\section{Caracterização da amostra}

Em 50\% dos casos da amostra, a movimentação da ponta da cúspide foi superior à movimentação da porção cervical da face vestibular, ou seja, houve um movimento de inclinação da coroa dos canino e, nos demais casos (50\%), a movimentação da ponta da cúspide foi igual ou inferior à da porção cervical da face vestibular, ocorrendo um movimento de translação dos caninos.
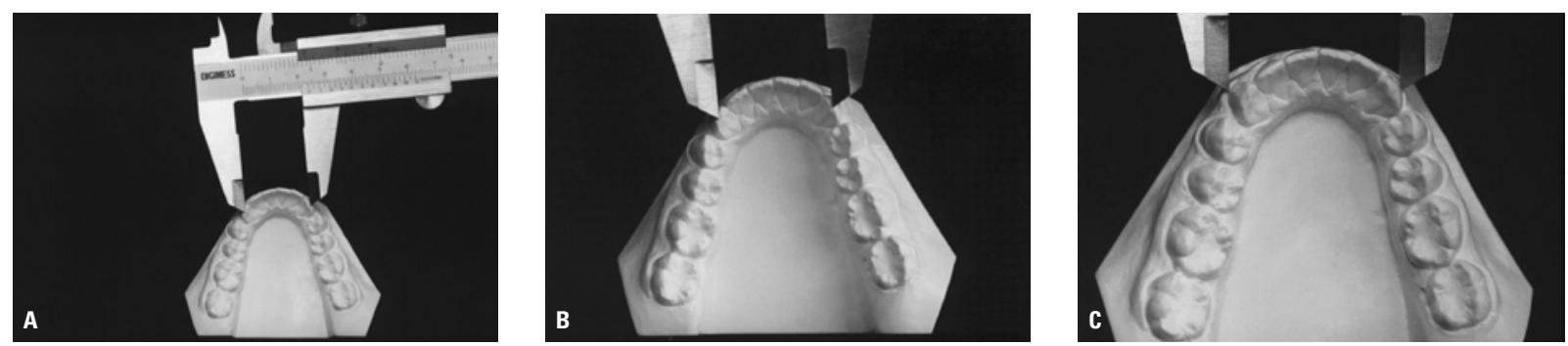

FIGURA 1 - A) Paquímetro utilizado para realizar as medições nos modelos de estudo. B) Distância intercaninos no arco mandibular com referência na ponta de cúspide. C) Distância intercaninos no arco mandibular com referência na face vestibular. 
Foi verificado que o percentual de casos com movimento de inclinação dos caninos foi significantemente inferior no grupo de pacientes em que foi feita a extração (25\%), quando comparado com o grupo sem extração $(66,7 \%)$. Esses resultados podem ser verificados no gráfico 2 .

\section{Alterações observadas na distância intercani- nos}

Como pode ser observado no gráfico 3 e na tabela 3, houve um aumento significante da distância intercaninos da primeira para a segunda avaliação (T2-T1), tanto com referência na ponta da cúspide quanto na face vestibular. Comparando-se os valores pós-tratamento (T2) e pós-contenção (T3), observou-se um decréscimo significante, sendo que na ponta da cúspide a distância intercaninos igualou-se com a primeira avaliação $(\mathrm{T} 3=\mathrm{T} 1)$ e na face vestibular a terceira avaliação manteve-se superior à primeira avaliação (T3>T1).

Os valores médios da distância intercaninos, na ponta da cúspide, foram $26,06 \pm 1,48 \mathrm{~mm}$, em $\mathrm{T} 1,26,92 \pm 1,33 \mathrm{~mm}$ em T2 e $26,23 \pm 1,42 \mathrm{~mm}$ em T3. Na face vestibular, mediu-se $29,80 \pm 1,61 \mathrm{~mm}$ em T1, 31,08 $\pm 1,78 \mathrm{~mm}$ em T2 e $30,79 \pm 1,67 \mathrm{~mm}$, em T3.

\section{Alterações na distância intercaninos inferior: comparação dos subgrupos}

\section{a) Gênero feminino e masculino}

Comparando-se o grupo de pacientes do gênero feminino com os do gênero masculino, em relação às alterações na distância intercaninos durante o tratamento (T2-T1), no período pós-contenção (T3-T2) e no período total avaliado (T3-T1), não se verificou diferenças significantes estatisticamente, como pode ser observado no gráfico 4 e na tabela 4.

Ao avaliar as alterações ocorridas na ponta da cúspide, observa-se que, nos pacientes do gênero feminino, houve uma expansão de $0,69 \pm 1,47 \mathrm{~mm}$, durante o tratamento (T2-T1), uma redução de
0,71 $\pm 0,74 \mathrm{~mm}$ no pós-tratamento (T3-T2) e uma redução de $0,02 \pm 1,49 \mathrm{~mm}$ no período total observado (T3-T1). Nos pacientes do gênero masculino, houve uma expansão de $1,41 \pm 1,16 \mathrm{~mm}$, de T1 para $\mathrm{T} 2$, uma redução de $0,61 \pm 0,48 \mathrm{~mm}$, após o tratamento (T3-T2), e um ganho total (T3-T1) de $0,80 \pm 1,29 \mathrm{~mm}$.

A movimentação ocorrida na porção cervical da face vestibular também não foi estatisticamente diferente entre homens e mulheres. No período do tratamentoortodôntico(T2-T1), houveumaexpansão de $1,30 \pm 1,15 \mathrm{~mm}$ e de $1,27 \pm 1,65 \mathrm{~mm}$, respectivamente. Após o término do tratamento (T3-T2),

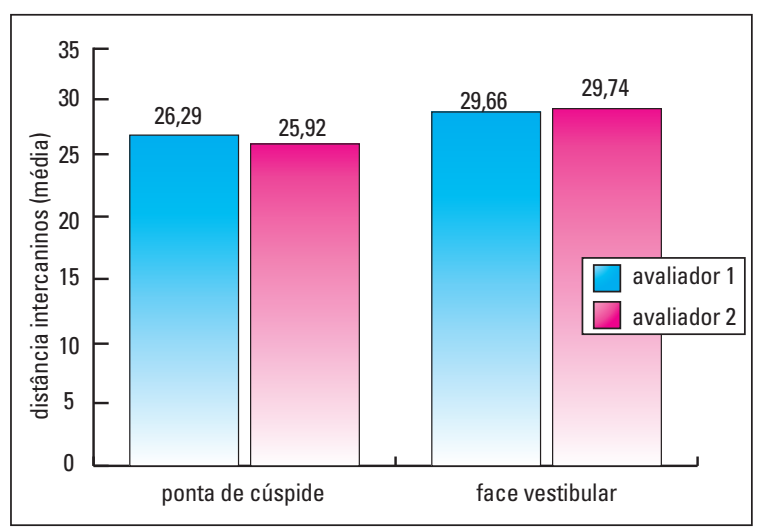

GRÁFICO 1 - Comparação entre dois avaliadores quanto à distância intercaninos com referência na ponta de cúspide e na face vestibular.

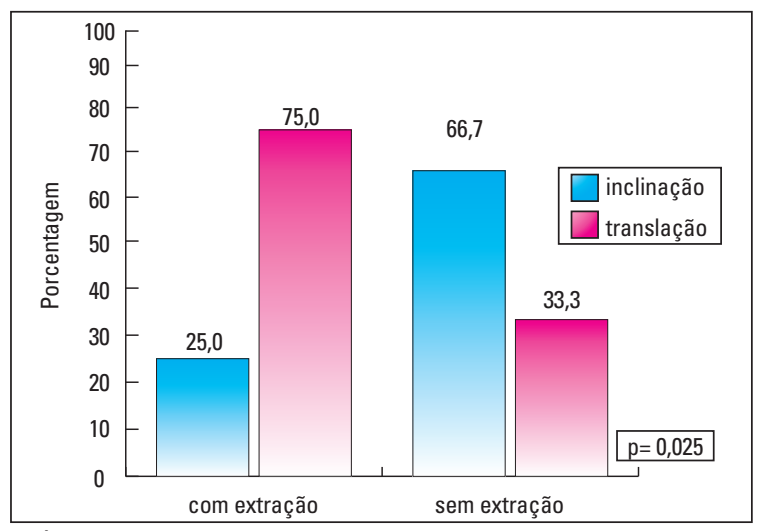

GRÁFICO 2 - Distribuição dos pacientes segundo o tipo de movimento realizado nos caninos, considerando-se a extração de pré-molares no tratamento.

Nota: A probabilidade de significância refere-se ao teste Qui-quadrado. 
Tabela 3 - Avaliação da distância intercaninos inferior nos três períodos (T1, T2 e T3), considerando-se a ponta da cúspide e a face vestibular como pontos de referência.

\begin{tabular}{|c|c|c|c|c|c|c|c|}
\hline \multicolumn{8}{|c|}{ medidas descritivas } \\
\hline referência & avaliação & mínimo & máximo & mediana & média & desvio-padrão & $\mathbf{p}$ \\
\hline \multirow{3}{*}{$\begin{array}{l}\text { ponta de } \\
\text { cúspide }\end{array}$} & T1 & 22,65 & 28,35 & 26,40 & 26,06 & 1,48 & \multirow{3}{*}{$<0,001^{*}$} \\
\hline & $\mathrm{T} 2$ & 22,50 & 29,00 & 27,00 & 26,92 & 1,33 & \\
\hline & T3 & 22,40 & 28,90 & 26,27 & 26,23 & 1,42 & \\
\hline \multirow{3}{*}{$\begin{array}{c}\text { face } \\
\text { vestibular }\end{array}$} & $\mathrm{T} 1$ & 25,90 & 32,45 & 30,12 & 29,80 & 1,61 & \multirow{3}{*}{$<0,001^{*}$} \\
\hline & $\mathrm{T} 2$ & 27,00 & 34,60 & 31,35 & 31,08 & 1,78 & \\
\hline & T3 & 26,40 & 34,30 & 30,92 & 30,79 & 1,67 & \\
\hline
\end{tabular}

* Diferenças estatisticamente significantes de acordo com o teste de Friedman.

T1- Pré-tratamento; T2- Pós-tratamento; T3- Período após remoção das contenções.

Tabela 4 - Avaliação da influência do gênero nas alterações da distância intercaninos inferior.

\begin{tabular}{|c|c|c|c|c|c|c|c|c|}
\hline referência & medida & gênero & mínimo & máximo & mediana & média & desvio-padrão & p \\
\hline \multirow{6}{*}{$\begin{array}{l}\text { ponta de } \\
\text { cúspide }\end{array}$} & \multirow{2}{*}{$\mathrm{T} 2$ - T1 } & fem. & $-2,60$ & 4,00 & 0,75 & 0,69 & 1,47 & \multirow{2}{*}{0,229} \\
\hline & & masc. & 0,20 & 3,80 & 1,10 & 1,41 & 1,16 & \\
\hline & \multirow{2}{*}{$\mathrm{T} 3$ - T2 } & fem. & $-2,40$ & 1,0 & $-0,75$ & $-0,71$ & 0,74 & \multirow{2}{*}{0,922} \\
\hline & & masc. & $-1,20$ & 0,10 & $-0,60$ & $-0,61$ & 0,48 & \\
\hline & \multirow{2}{*}{ T3 - T1 } & fem. & $-3,20$ & 3,10 & $-0,05$ & $-0,02$ & 1,49 & \multirow{2}{*}{0,162} \\
\hline & & masc. & $-0,75$ & 3,20 & 0,80 & 0,80 & 1,29 & \\
\hline \multirow{6}{*}{$\begin{array}{c}\text { face } \\
\text { vestibular }\end{array}$} & \multirow{2}{*}{ T2 - T1 } & fem. & $-2,10$ & 4,25 & 1,15 & 1,27 & 1,65 & \multirow{2}{*}{0,922} \\
\hline & & masc. & 0,15 & 3,60 & 0,95 & 1,30 & 1,15 & \\
\hline & \multirow{2}{*}{ T3 - T2 } & fem. & $-1,35$ & 0,75 & $-0,15$ & $-0,26$ & 0,56 & \multirow{2}{*}{0,606} \\
\hline & & masc. & $-1,20$ & 0,35 & $-0,15$ & $-0,37$ & 0,55 & \\
\hline & \multirow{2}{*}{ T3 - T1 } & fem. & $-1,35$ & 4,65 & 0,90 & 1,02 & 1,41 & \multirow{2}{*}{0,695} \\
\hline & & masc. & $-0,80$ & 3,95 & 0,65 & 0,93 & 4,59 & \\
\hline
\end{tabular}

Não houve diferença estatisticamente significante de acordo com o teste de Kruskal-Wallis.

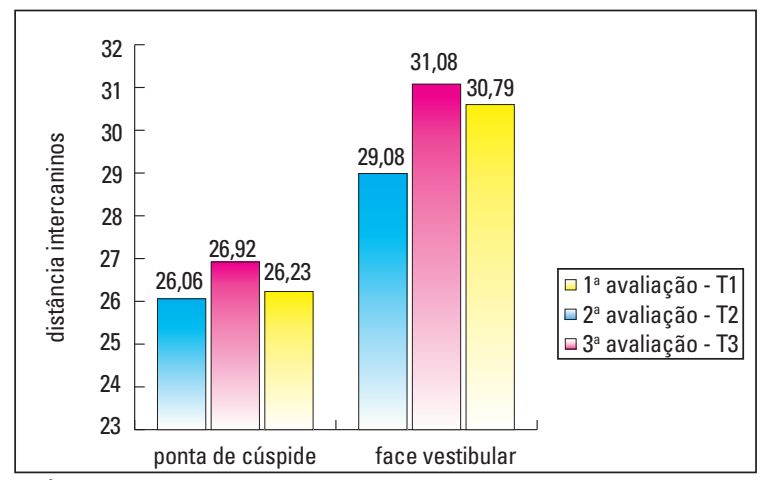

GRÁFICO 3 - Gráfico com a distância intercaninos inferior em T1, T2 e T3.

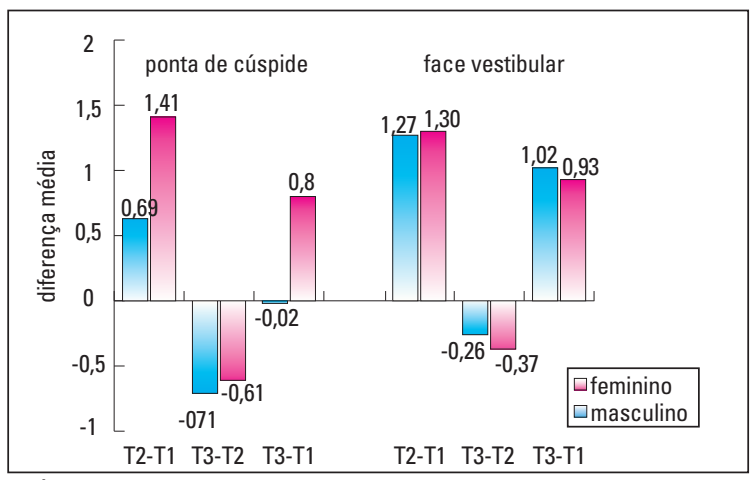

GRÁFICO 4 - Gráfico com a avaliação da influência do gênero nas diferenças observadas entre as três avaliações. 
houve uma redução da distância intercaninos de $0,37 \pm 0,55 \mathrm{~mm}$ nos homens e de $0,26 \pm 0,56 \mathrm{~mm}$ nas mulheres. No período total avaliado (T3-T1), houve um ganho de $0,93 \pm 4,59 \mathrm{~mm}$ nos homens e de $1,02 \pm 1,41 \mathrm{~mm}$, nas mulheres.

b) Tratamento com e sem extração de pré-molares

O gráfico 5 e a tabela 5 mostram a influência da extração de pré-molares. Considerando-se os valores obtidos com referência na ponta da cúspide, pode-se observar que a redução da distância

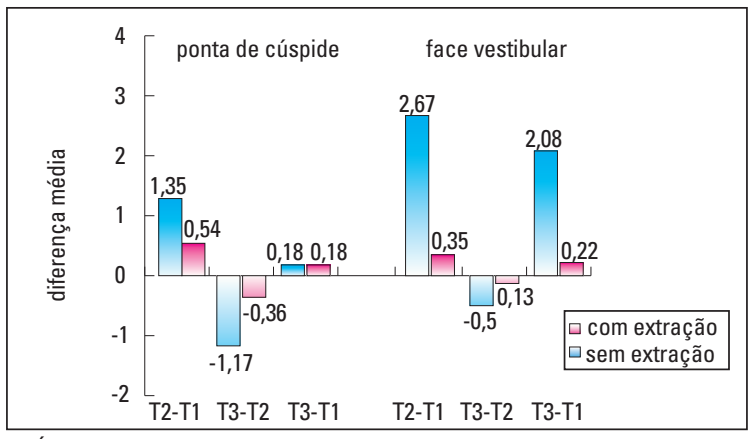

GRÁFICO 5 - Gráfico com a avaliação da influência da extração de pré-molares nas alterações da distância intercaninos inferior. intercaninos entre T3 e T2 foi significantemente maior nos casos com extração $(-1,17 \pm 0,55 \mathrm{~mm})$ do que nos casos sem extração $(-0,36 \pm 0,56 \mathrm{~mm})$. A expansão ocorrida durante o tratamento (T2T1) e o ganho total (T3-T1) não foram estatisticamente diferentes entre os grupos. No grupo com extração, observou-se uma expansão média de $1,35 \pm 1,46 \mathrm{~mm}$ durante o tratamento (T2-T1) e uma expansão total, ao final das observações (T3$\mathrm{T} 1$ ), de $0,18 \pm 1,67 \mathrm{~mm}$. Já o grupo tratado sem extração obteve uma expansão média, durante o tratamento (T2-T1), de $0,54 \pm 1,34 \mathrm{~mm}$ e um ganho total (T3-T1) de $0,18 \pm 1,37 \mathrm{~mm}$.

Já em relação à face vestibular, considerandose as diferenças entre T2 e Tl (alterações durante o tratamento) e entre T3 e Tl (alterações totais), verificaram-se valores significantemente superiores no grupo em que houve extração, revelando que houve maior expansão durante o tratamento e que o ganho total, ao final das observações, foi significantemente maior que no grupo tratado sem extração. Os valores observados no grupo com extração foram 2,67 $\pm 1,03 \mathrm{~mm}$ de expansão (T2-T1) e 2,08 $\pm 1,18 \mathrm{~mm}$ de ganho total (T3-T1). No grupo tratado sem extração, observou-se uma expansão

Tabela 5 - Avaliação da influência da extração de pré-molares nas alterações da distância intercaninos inferior.

\begin{tabular}{|c|c|c|c|c|c|c|c|c|}
\hline \multicolumn{9}{|c|}{ medidas descritivas } \\
\hline referência & medida & extração & mínimo & máximo & mediana & média & desvio-padrão & $\mathbf{p}$ \\
\hline \multirow{6}{*}{$\begin{array}{l}\text { ponta da } \\
\text { cúspide }\end{array}$} & \multirow{2}{*}{$\mathrm{T} 2-\mathrm{T} 1$} & $\operatorname{sim}$ & $-0,85$ & 4,00 & 0,80 & 1,35 & 1,46 & \multirow{2}{*}{0,374} \\
\hline & & não & $-2,60$ & 2,60 & 0,80 & 0,54 & 1,34 & \\
\hline & \multirow{2}{*}{ T3 - T2 } & $\operatorname{sim}$ & $-2,40$ & $-0,55$ & $-0,93$ & $-1,17$ & & \multirow{2}{*}{$0,002^{*}$} \\
\hline & & não & $-1,20$ & 1,00 & $-0,48$ & $-0,36$ & 0,56 & \\
\hline & \multirow{2}{*}{ T3 - T1 } & $\operatorname{sim}$ & $-2,35$ & 3,20 & 0,00 & 0,18 & 1,67 & \multirow{2}{*}{0,783} \\
\hline & & não & $-3,20$ & 2,45 & 0,23 & 0,18 & 1,37 & \\
\hline \multirow{6}{*}{$\begin{array}{c}\text { face } \\
\text { vestibular }\end{array}$} & \multirow{2}{*}{$\mathrm{T} 2$ - T1 } & $\operatorname{sim}$ & 0,90 & 4,25 & 2,73 & 2,67 & 1,03 & \multirow{2}{*}{$<0,001^{*}$} \\
\hline & & não & $-2,10$ & 1,80 & 0,55 & 0,35 & 1,02 & \\
\hline & \multirow{2}{*}{$\mathrm{T} 3-\mathrm{T} 2$} & $\operatorname{sim}$ & $-1,35$ & 0,40 & $-0,43$ & $-0,50$ & 0,59 & \multirow{2}{*}{0,079} \\
\hline & & não & $-1,20$ & 0,75 & $-0,05$ & $-0,13$ & 0,48 & \\
\hline & \multirow{2}{*}{ T3 - T1 } & $\operatorname{sim}$ & 0,60 & 4,65 & 1,90 & 2,08 & 1,18 & \multirow{2}{*}{$<0,001^{*}$} \\
\hline & & não & $-1,35$ & 1,65 & 0,58 & 0,22 & 0,97 & \\
\hline
\end{tabular}

* Diferenças estatisticamente significantes de acordo com o teste de Kruskal-Wallis. 
durante o tratamento (T2-T1) de $0,35 \pm 1,02 \mathrm{~mm}$ e um ganho total (T3-T1) de $0,22 \pm 0,97 \mathrm{~mm}$. É interessante observar que a redução da distância intercaninos, no período pós-tratamento (T3-T2), não foi estatisticamente diferente entre os grupos. Os pacientes tratados com extração sofreram uma redução de $0,50 \pm 0,59 \mathrm{~mm}$, já os pacientes tratados sem extração sofreram uma redução de $0,13 \pm 0,48 \mathrm{~mm}$.

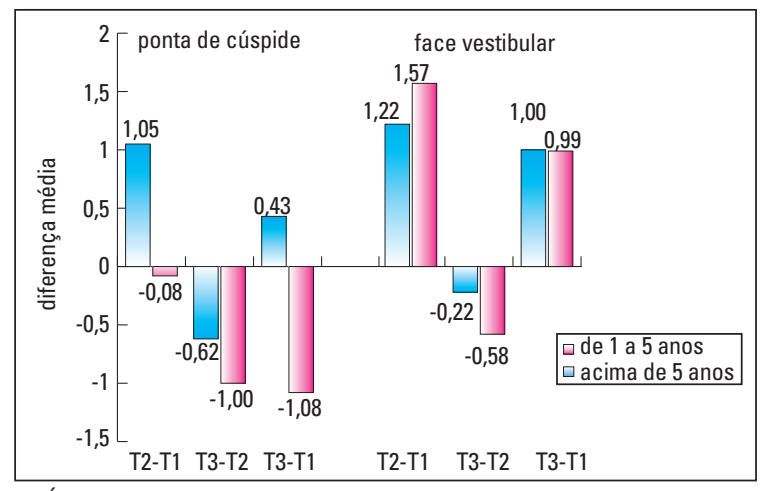

GRÁFICO 6 - Gráfico com a avaliação da influência do tempo pós-contenção nas alterações da distância intercaninos inferior. c) Ausência de uso da contenção de um a cinco anos e acima de cinco anos

Como pode ser observado no gráfico 6 e na tabela 6 , não foram observadas diferenças significantes entre o grupo com tempo sem contenção entre um e cinco anos e aquele com mais de cinco anos, no que diz respeito às alterações na distância intercaninos, em todos os períodos avaliados e nas duas referências utilizadas (ponta da cúspide e região cervical da face vestibular).

No grupo de um a cinco anos sem contenção, considerando-se o movimento realizado na ponta da cúspide, houve uma expansão de $1,05 \pm 1,31 \mathrm{~mm}$ durante o tratamento (T2-T1), seguida de uma constrição de $0,62 \pm 0,71 \mathrm{~mm}$ no período pós-tratamento (T3-T2). O ganho total foi de $0,43 \pm 1,36 \mathrm{~mm}$ em todo período observado (T3-T1). No grupo com mais de cinco anos sem uso de contenção inferior, a alteração durante o tratamento (T2-T1) foi de $-0,08 \pm 1,72 \mathrm{~mm}$, de $-1,00 \pm 0,47 \mathrm{~mm}$ após o tratamento (T3-T2), e de $-1,08 \pm 1,49 \mathrm{~mm}$ no período total de observação (T3-T1).

Os valores obtidos na região cervical da face

Tabela 6 - Avaliação da influência do tempo pós-contenção nas alterações da distância intercaninos inferior.

\begin{tabular}{|c|c|c|c|c|c|c|c|c|}
\hline \multicolumn{9}{|c|}{ medidas descritivas } \\
\hline referência & medida & tempo & mínimo & máximo & mediana & média & desvio-padrão & $\mathbf{p}$ \\
\hline \multirow{6}{*}{$\begin{array}{l}\text { ponta de } \\
\text { cúspide }\end{array}$} & \multirow{2}{*}{$\mathrm{T} 2-\mathrm{T} 1$} & 1 a 5 & $-2,10$ & 4,00 & 0,85 & 1,05 & 1,31 & \multirow{2}{*}{0,597} \\
\hline & & $>5$ & $-2,60$ & 1,95 & 0,50 & $-0,08$ & 1,72 & \\
\hline & \multirow{2}{*}{$\mathrm{T} 3-\mathrm{T} 2$} & 1 a 5 & $-2,40$ & 1,00 & $-0,70$ & $-0,62$ & 0,71 & \multirow{2}{*}{0,148} \\
\hline & & $>5$ & $-1,70$ & $-0,55$ & $-0,95$ & $-1,00$ & 0,47 & \\
\hline & \multirow{2}{*}{ T3 - T1 } & 1 a 5 & $-2,35$ & 3,20 & 0,45 & 0,43 & 1,36 & \multirow{2}{*}{0,200} \\
\hline & & $>5$ & $-3,20$ & 0,25 & $-0,45$ & $-1,08$ & 1,49 & \\
\hline \multirow{6}{*}{$\begin{array}{c}\text { face } \\
\text { vestibular }\end{array}$} & \multirow{2}{*}{$\mathrm{T} 2-\mathrm{T} 1$} & 1 a 5 & $-2,10$ & 4,25 & 1,10 & 1,22 & 1,49 & \multirow{2}{*}{0,080} \\
\hline & & $>5$ & $-1,25$ & 3,30 & 1,65 & 1,57 & 1,89 & \\
\hline & \multirow{2}{*}{ T3 - T2 } & 1 a 5 & $-1,20$ & 0,75 & $-0,15$ & $-0,22$ & 0,53 & \multirow{2}{*}{0,578} \\
\hline & & $>5$ & $-1,35$ & 0,05 & $-0,55$ & $-0,58$ & 0,62 & \\
\hline & \multirow{2}{*}{$\mathrm{T} 3-\mathrm{T} 1$} & 1 a 5 & $-1,35$ & 4,65 & 0,90 & 1,00 & 1,44 & \multirow{2}{*}{0,373} \\
\hline & & $>5$ & $-1,20$ & 2,75 & 0,90 & 0,99 & 1,49 & \\
\hline
\end{tabular}

Não houve diferença estatisticamente significante de acordo com o teste de Kruskal-Wallis.

$\mathrm{G} 1$ = de 1 a 5 anos sem uso de contenção; $\mathrm{G} 2$ = acima de 5 anos sem uso de contenção. 
Avaliação das alterações na distância intercaninos do arco inferior em pacientes com má oclusão de Classe I tratados ortodonticamente

Tabela 7 - Avaliação da influência do tipo de movimento realizado nos caninos sobre as alterações na distância intercaninos inferior.

\begin{tabular}{|c|c|c|c|c|c|c|c|c|}
\hline \multicolumn{9}{|c|}{ Medidas descritivas } \\
\hline referência & medida & grupo & mínimo & máximo & mediana & média & desvio-padrão & p \\
\hline \multirow{4}{*}{$\begin{array}{l}\text { ponta de } \\
\text { cúspide }\end{array}$} & \multirow{2}{*}{$\mathrm{T} 3-\mathrm{T} 2$} & 1 & $-1,75$ & 0,45 & $-0,50$ & $-0,51$ & 0,56 & \multirow{2}{*}{0,051} \\
\hline & & 2 & $-2,40$ & 1,00 & $-0,90$ & $-0,87$ & 0,76 & \\
\hline & \multirow{2}{*}{$\mathrm{T} 3$ - T1 } & 1 & $-1,45$ & 3,20 & 1,00 & 0,97 & 1,35 & \multirow{2}{*}{$0,003^{*}$} \\
\hline & & 2 & $-3,20$ & 0,80 & $-0,40$ & $-0,62$ & 1,14 & \\
\hline \multirow{4}{*}{$\begin{array}{c}\text { face } \\
\text { vestibular }\end{array}$} & \multirow{2}{*}{$\mathrm{T} 3-\mathrm{T} 2$} & 1 & $-1,20$ & 0,75 & $-0,15$ & $-0,24$ & 0,59 & \multirow{2}{*}{0,787} \\
\hline & & 2 & $-1,35$ & 0,40 & $-0,15$ & $-0,33$ & 0,52 & \\
\hline & \multirow{2}{*}{ T3 - T1 } & 1 & $-1,35$ & 3,95 & 0,65 & 0,56 & 1,38 & \multirow{2}{*}{$0,038^{*}$} \\
\hline & & 2 & $-1,20$ & 4,65 & 1,50 & 1,43 & 1,38 & \\
\hline
\end{tabular}

*Diferenças estatisticamente significantes de acordo com o teste de Kruskal-Wallis.

Grupo 1= Movimento de Inclinação; Grupo 2= Movimento de Translação.

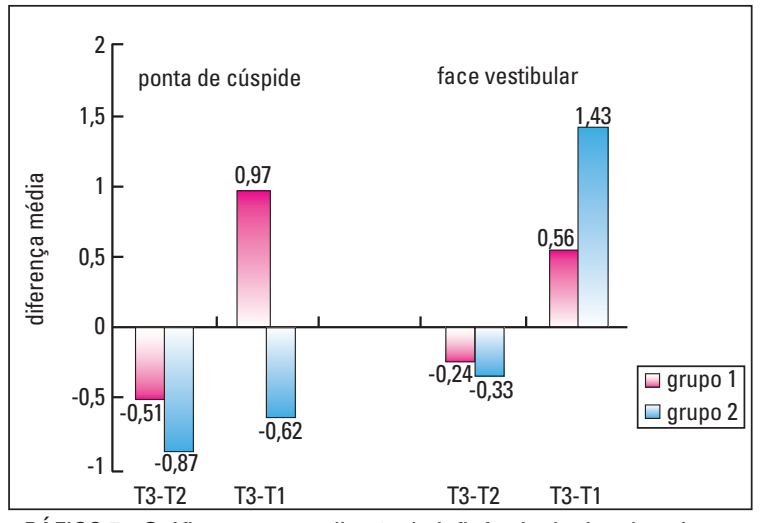

GRÁFICO 7 - Gráfico com a avaliação da influência do tipo de ovimento realizado nos caninos sobre as alterações na distância intercaninos inferior.

Nota: Grupo 1 = Movimento de Inclinação; Grupo 2 = Movimento de Translação.

vestibular nos grupos de um a cinco anos e acima de cinco anos sem contenção foram, respectivamente, $1,22 \pm 1,49 \mathrm{~mm}$ e $1,57 \pm 1,89 \mathrm{~mm}$ de expansão, durante o tratamento (T2-T1), $-0,22 \pm 0,53 \mathrm{~mm}$ e $-0,58 \pm 0,62 \mathrm{~mm}$ de redução, no

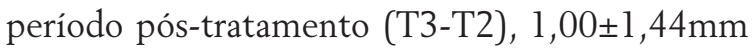
e $0,99 \pm 1,49 \mathrm{~mm}$ de ganho total, ao final das observações (T3-T1).

d) Tipo de movimento realizado nos caninos: translação $X$ inclinação

Comparando-se a quantidade de movimento da ponta da cúspide com a quantidade de movimento da face vestibular (região cervical), pôdese dividir os pacientes em dois grupos, de acordo com o tipo de movimento realizado nos caninos durante o tratamento.

No grupo 1 foram colocados aqueles pacientes nos quais o movimento da ponta da cúspide foi maior que o da face vestibular, ocorrendo, então, um movimento de inclinação vestibular dos caninos. Já no grupo 2 incluíram-se os pacientes nos quais o movimento da face vestibular foi igual ao da ponta da cúspide, considerando-se, então, que houve um movimento de translação para vestibular dos caninos.

Observou-se que o grupo 1 apresentou resultados significantemente inferiores de recidiva póstratamento (T3-T1) em relação ao grupo 2, quando a referência foi a ponta da cúspide. Entretanto, quando a referência utilizada foi a face vestibular, o grupo 2 apresentou uma maior estabilidade da expansão em relação ao grupo 1 .

O grupo 1 obteve um ganho médio de $0,97 \mathrm{~mm}$ na ponta da cúspide e de $0,56 \mathrm{~mm}$ na região cervical da face vestibular. O grupo 2 obteve uma redução de $0,62 \mathrm{~mm}$, em média, na ponta da cúspide e um ganho médio de $1,43 \mathrm{~mm}$ na região cervical da face vestibular dos caninos (Gráf. 7, Tab. 7). 


\section{DISCUSSÃO}

Avaliações acerca da imutabilidade da distância intercaninos no tratamento ortodôntico têm sido um tema constante na literatura ortodôntica. Horowitz e Hixon ${ }^{22}$ afirmaram que o ponto significativo é que a terapia ortodôntica pode alterar, temporariamente, o curso das mudanças fisiológicas contínuas e, possivelmente, até revertê-las por algum tempo; entretanto, após a mecanoterapia e o período de contenção, o processo de maturação retorna. Portanto, é necessário que o ortodontista tenha conhecimento do curso dessas alterações, para poder planejar contenções adequadas e conscientizar seus pacientes das limitações quanto à estabilidade, em longo prazo, de um tratamento ortodôntico realizado com expansão da distância intercaninos.

No presente trabalho, a escolha de apenas um tipo de má oclusão se deu no intuito de homogeneizar, dentro do possível, o tipo de tratamento instituído. Alguns autores, como Little, Wallen, Riedel ${ }^{24}$ e Burke et al. $^{10}$, não encontraram diferença na estabilidade da distância intercaninos entre as diferentes classes de má oclusão de Angle. Entretanto, Shapiro ${ }^{40}$ observou que os pacientes Classe II, 2a divisão, possuem maior capacidade de manter a expansão da distância intercaninos conseguida no tratamento ortodôntico. Assim, a escolha de pacientes com má oclusão Classe I de Angle como amostra deste estudo objetivou uma comparação mais fiel dos dados obtidos.

Os pacientes deste estudo demonstraram um aumento da distância intercaninos, durante o tratamento, e uma redução no período pós-tratamento. Esses resultados estão de acordo com outros estudos ${ }^{1,8,10,14,15,19,23,26,28,49}$.

Essa tendência à recidiva foi observada tanto com relação à ponta da cúspide quanto à face vestibular. Entretanto, quando se utilizou a face vestibular como referência, observou-se que alguma expansão foi mantida. Pinto ${ }^{33}$, ao comparar as alterações ocorridas na distância intercaninos, também observou que parte da expansão ocorrida no nível da margem gengival, na face vestibular dos caninos, foi mantida no período pós-contenção. Este autor relata também que a redução ocorrida nas pontas das cúspides levou a um retorno da distância intercaninos para valores ligeiramente abaixo dos originais.

As diferenças nas alterações da distância intercaninos, durante e depois do tratamento, entre os gêneros, não se mostraram estatisticamente significantes. Esse achado está de acordo com os estudos de Moorrees, Chada ${ }^{31}$; Bishara, Bayati, Zaher, Jakobsen $^{4}$; Carter, McNamara Jr. ${ }^{11}$ e Burke et al. ${ }^{10}$

Ao se comparar os pacientes tratados com extração de pré-molares e os pacientes tratados sem extração, pode-se perceber que existiu uma diferença estatisticamente significante na quantidade de recidiva da distância intercaninos inferior, de T3 para T2, medida na ponta da cúspide, sendo que essa recidiva foi maior no grupo tratado com extrações. Porém, comparando-se com as alterações ocorridas na porção gengival da face vestibular, observa-se que os casos tratados com extrações foram, significantemente, mais expandidos durante o tratamento que os casos tratados sem extrações. Além disso, o ganho real, ao final das observações (T3-T1), também foi significantemente maior nos pacientes tratados com extrações. Conclui-se, então, que a maior quantidade de recidiva ocorrida nos casos de extrações tenha sido decorrente da maior quantidade de expansão obtida no tratamento. Esse achado está de acordo com o estudo de Sondhi, Cleall e Bego$1 \mathrm{e}^{44}$. Eles concluíram que o maior grau de recidiva dos casos com movimentação distal dos caninos para o espaço das extrações estava relacionado à maior expansão realizada no tratamento. Já os estudos de Kaplan ${ }^{23}$; Rossouw, Preston ${ }^{37}$; Bishara, Bayati, Zaher e Jakobsen ${ }^{4}$ relatam não haver diferenças entre os pacientes tratados com ou sem extrações, com relação à quantidade de recidiva da distância intercaninos inferior.

Os resultados dos pacientes com tempos diferentes sem uso de contenção inferior não re- 
velaram diferenças estatisticamente significantes. De acordo com o estudo de Vaden e Gardner ${ }^{50}$, a maior parte da recidiva da distância intercaninos ocorre logo após o tratamento. No presente estudo, o fato de o grupo de pacientes fora de contenção há mais de cinco anos ter sido tratado com movimento de translação dos caninos, e apenas $40 \%$ do grupo de um a cinco anos sem contenção ter tido esse tipo de movimento, não resultou em diferenças significantes da recidiva pós-tratamento da distância intercaninos. Porém, o grupo de pacientes há mais de cinco anos sem uso da contenção inferior é muito pequeno (cinco casos). Talvez, aumentando-se a quantidade de pacientes da amostra, o resultado fosse diferente.

Ao se relacionar o tipo de movimento realizado nos caninos com a ocorrência de recidiva da expansão, no período pós-tratamento (T3-T2), não foram observadas diferenças estatisticamente significantes entre os grupos de translação e de inclinação. Entretanto, se forem analisadas as alterações ocorridas no tempo total entre as avaliações (T3-T1), se verificará que os casos de translação dos caninos se mostraram mais estáveis quando a referência utilizada foi a face vestibular e menos estáveis quando a referência foi a ponta da cúspide. Isso pode significar que os dentes expandidos com movimento de translação sofreram alguma verticalização durante o tratamento e apresentaram uma tendência a inclinarem-se lingualmente, no período pós-contenção. Não foram encontrados, na literatura, estudos que avaliassem o tipo de movimento realizado na expansão dos caninos e a recidiva da distância intercaninos, portanto, não foi possível comparar os resultados obtidos neste trabalho com os de outros autores.

$\mathrm{Na}$ caracterização dos pacientes de acordo com o tipo de movimento realizado na expansão dos caninos, pôde-se observar uma diferença nos resultados dos grupos tratados com e sem extração de pré-molares. No grupo tratado sem extração, o percentual de pacientes que teve inclinação dos caninos $(66,7 \%)$ foi muito superior ao percentual observado nos pacientes tratados com extração $(25 \%)$. Esse fato talvez seja explicado pela distalização dos caninos para o local da extração. Conseqüentemente, a expansão da distância intercaninos ocorre mais pela localização dos caninos em um local mais amplo do arco, do que pela inclinação vestibular desses dentes.

A aplicação clínica desses resultados refere-se ao aconselhamento apropriado dos pacientes no período pós-tratamento. Ao considerar o aumento da distância intercaninos inferior no tratamento ortodôntico, o profissional deve estar consciente de que uma alta percentagem dos pacientes tratados com esse procedimento irá apresentar uma redução, no período pós-contenção, a valores próximos aos inicias. Sendo assim, uma contenção em longo prazo ou até permanente pode ser mandatória.

Este trabalho se limitou ao estudo das alterações na distância intercaninos inferior no tratamento ortodôntico e no período pós-contenção. Seria interessante avaliar também, em estudos futuros, a estabilidade da expansão na região de pré-molares, a relação entre os tipos faciais e a distância intercaninos e entre esta e a forma do arco dentário.

\section{CONCLUSÕES}

Nos pacientes avaliados neste estudo, observou-se que:

1) Durante o tratamento ortodôntico, houve um aumento da distância intercaninos inferior, independente do gênero e da realização ou não de extrações de pré-molares.

2) No período pós-contenção, houve uma redução da distância intercaninos inferior, independente do gênero, da realização ou não de extrações de pré-molares, do tempo sem uso da contenção inferior e do tipo de movimento realizado nos caninos durante o tratamento.

3) A distância intercaninos mostrou capacidade de manter alguma parte da expansão conseguida, 
quando medida na face vestibular dos caninos.

4) A redução da distância intercaninos inferior ocorreu de forma indiferenciada nos pacientes com diferentes períodos fora de contenção, indicando que a maioria das alterações ocorre logo após a remoção das contenções.

5) A distância intercaninos inferior dos casos tratados com extração de pré-molares foi mais expandida durante o tratamento e teve um ganho real, ao final das observações, maior que os casos tratados sem extração, de acordo com as medidas feitas na face vestibular.

Enviado em: janeiro de 2004 Revisado e aceito: setembro de 2004

\title{
Intercanine width changes in the mandibular arch of Class I malocclusion patients orthodontically treated
}

\begin{abstract}
Introduction: Intercanine width changes in the mandibular arch during orthodontic treatment are considered unstable. Aim and Methods: In order to observe the post treatment alterations in the lower intercanine width, 30 patients with Class I malocclusion were selected from the files of the graduate orthodontic clinic of the Catholic University of Minas Gerais based on the availability of records. The measurements were made on study models, taken in two areas (cuspid tip and buccal surface) and in three different periods of time: T1 (pre treatment); T2 (post treatment) and T3 (at least one year out of retention). Results and Conclusions: There was a significant increase in the intercanine width during treatment and a reduction in the post retention period at the cuspid tip and the buccal surface. When the area of measurement was considered, there was no difference between T3 and T1 at the cuspid tip. At buccal surface it was significantly greater in T3 than in T13. There were no statistical differences in the intercanine width related to gender and time out of retention. The extraction group showed a significantly greater increase in the intercanine width than the non extraction group in the cuspid tip and in the buccal surface. The extraction group showed a significant greater reduction of the intercanine width measured at the cuspid tip when compared to measurements at the buccal surface. The extraction cases were more stable than the non extraction cases considering the buccal surface. Considering the type of movement used for canine expansion, the translation cases were more stable with measurements at buccal surface than at cuspid tip, indicating a lingual inclination of the crowns of the canines in the post retention period.
\end{abstract}

Key words: Intercanine width. Expansion. Relapse.

\section{REFERÊNCIAS}

1. ADES, A. G.; JOONDEPH, D. R.; LITTLE, R. M.; CHAPKO, M. K. A long-term study of the relationship of third molars to changes in the mandibular dental arch. Am J Orthod Dentofacial Orthop, St. Louis, v. 97, no. 4, p. 323-335, Apr. 1990.

2. ANGLE, E. Treatment of malocclusion of the teeth. Angle's system. 7th ed. Philadelphia: S. S. White Manufacturing, 1907.

3. BARROW, C. V.; WHITE, J. R. Developmental changes of the maxilary and mandibular dental arches. Angle Orthod, Appleton, v. 22, no. 1, p. 41-46, Jan. 1952.

4. BISHARA, S. E.; BAYATI, P.; ZAHER, A. R.; JAKOBSEN, J. R. Comparisons of the dental arch changes in patients with Class II, division 1 malocclusions: extraction vs nonextraction treatments. Angle Orthod, Appleton, v. 64, no. 5, p. 351-358, 1994.

5. BISHARA, S. E.; TREDER, J. E.; JAKOBSEN, J. R. Facial and dental changes in adulthood. Am J Orthod Dentofacial Orthop, St. Louis, v. 106, no. 2, p. 175-186, Aug. 1994.

6. BISHARA, S. E.; TREDER, J. E.; DAMON, P.; OLSEN, M. Changes in the dental arches and dentition between 25 and 45 years of age. Angle Orthod, Appleton, no. 6, p. 417-422, 1996.
7. BISHARA, S. E.; JAKOBSEN, J. R.; TREDER, J.; NOWAK, A. Arch width changes from 6 weeks to 45 years of age. Am J Orthod Dentofacial Orthop, St. Louis, v. 111, no. 4, p. 401-409, Apr. 1997.

8. BOESE, L. R. Fiberotomy and reaproximation without lower retention: nine years in retrospective: part II. Angle Orthod, Appleton, v. 50, no. 3, p. 169-178, July 1980.

9. BONDEVIK, O. Changes in occlusion between 23 and 34 years. Angle Orthod, Appleton, v. 68, no. 1, p. 75-80, Feb. 1998.

10. BURKE, S. P.; SILVEIRA, A. M.; GOLDSMITH, L. J.; YANCEY, J. M.; VAN STEWART, A.; SCARFE, W. C. A meta-analysis of mandibular intercanine width in treatment and post retention. Angle Orthod, Appleton, v. 68, no. 1, p. 53-60, Feb. 1998.

11. CARTER, G. A.; McNAMARA JR., J. A. Longitudinal dental arch changes in adults. Am J Orthod Dentofacial Orthop, St. Louis, v. 114, no. 1, p. 88-99, July 1998.

12. DAVIS, L. M.; BeGOLE, E. A. Evaluation of orthodontic relapse using the cubic spline function. Am J Orthod Dentofacial Orthop, St. Louis, v. 113, no. 3, p. 300-306, Mar. 1998. 
13. DUGONI, S. A.: LEE, J. S. VARELA, J.: DUGONI, A. A. Early mixed dentition treatment: post retention evaluation of stability and relapse. Angle Orthod, Appleton, v. 65, no. 5, p. 311-320, 1995.

14. EL MANGOURY, N. H. Orthodontic relapse in subjects with varying degrees of anteroposterior and vertical displasia. Am J Orthod, St. Louis, v. 75, no. 5, p. 548-561, May 1979.

15. ELMS, T. N.; BUSCHANG, P. H.; ALEXANDER, R. G. Long-term stability of Class II, Division 1, nonextraction cervical face-bow therapy: I. Model analysis. Am J Orthod Dentofacial Orthop, St. Louis, v. 109, no. 3, p. 271-276, Mar. 1996.

16. FASTLICHT, J. Crowding of the mandibular incisors. Am J Orthod, St. Louis, p. 156-163, Aug. 1970

17. GARDNER, S.; CHACONAS, S. Post treatment and post retention changes following orthodontic therapy. Angle Orthod, Appleton, v. 46, no. 2, p. 151-161, Apr. 1976

18. GERMANE, N.; LINDAUER, S. J.; RUBENSTEIN, L. K.; REVERE JR., J. H.; ISAACSON, R. J. Increase in arch perimeter due to orthodontic. Am J Orthod Dentofacial Orthop, St. Louis, v. 100 , no. 5 , p. $421-427$, Nov. 1991

19. GLENN, G.; SINCLAIR, P. M.; ALEXANDER, R. G. Nonextraction orthodontic therapy: post treatment dental and skeletal stability. Am J Orthod Dentofacial Orthop, St. Louis, p. 321-328, Oct. 1987.

20. HARRIS, E. F. A longitudinal study of arch size and form in untreated adults. Am J Orthod Dentofacial Orthop, St. Louis, v. 111, no. 4, p. 419-427, Apr. 1997.

21. HERBERGER, R. J. Stability of mandibular intercuspid width after long periods of retention. Angle Orthod, Appleton, v. 51, no. 1, p. 78-83, Jan. 1981

22. HOROWITZ, S.; HIXON, E. Physiologic recovery following orthodontic treatment. Am J Orthod, St. Louis, v. 55, p. 1-4, 1969

23. KAPLAN, R. G. Mandibular third molars and post retention crowding. Am J Orthod, St. Louis, v. 66, no. 4, p. 411-430, Oct. 1974.

24. LITTLE, R.; WALLEN, T.; RIEDEL, R. Stability and relapse of mandibular anterior alignment- first pre-molar extraction cases treated by traditional Edgewise Orthodontics. Am J Orthod, St. Louis, v. 80, no. 4, p. 349-365, Oct. 1981.

25. LITTLE, R. M.; RIEDEL, R. A.; ARTUN, J. An evaluation of changes in mandibular anterior alignment from 10 to 20 years post retention. Am J Orthod Dentofacial Orthop, St. Louis, v. 93, no. 5, p. 423-428, May 1988

26. LITTLE, R. M.; RIEDEL, R. A.; STEIN, A. Mandibular arch length increase during the mixed dentition - post retention evaluation of stability and relapse. Am J Orthod Dentofacial Orthop, St. Louis, v. 97, no. 5, p. 393-404, May 1990.

27. LITTLE, R. M.; RIEDEL, R. A.; ENGST, E. D. Serial extraction of first premolars: post retention evaluation of stability and relapse. Angle Orthod, Appleton, v. 60, no. 4, p. 255-262, 1990

28. McREYNOLDS, D. C.; LITTLE, R. M. Mandibular second premolar extraction - post retention evaluation of stability and relapse. Angle Orthod, Appleton, no. 2, p. 133-144, 1991

29. MEREDITH, I. L.; HIGLEY, L. B. Relationships between dental arch widths and widths of the face and head. Am J Orthod, St. Louis, v. 37, p. 193-204, 1951.

30. MIYAZAKI, H.; MOTEGI, E.; YATABE, K.; ISSHIKI, Y. Occlusal stability after extraction orthodontic therapy in adult and adolescent patients. Am J Orthod Dentofacial Orthop, St. Louis, v. 114 , no. 5 , p. 530-537, Nov. 1998

31. MOORREES, C. F. A.; CHADHA, M. J. Available space to the incisors during dental development. Angle Orthod, Appleton, v. 35, no. 1, p. 12-22, Jan. 1965

32. PEAK, J. D. Cuspid stability. Am J Orthod, St. Louis, v. 42, no. 8, p. 608-614, Aug. 1956.

33. PINTO, E. M. Estudo comparativo das distâncias intercaninos e intermolares em pacientes de Classe I e II, tratados ortodonticamente. 1981. 111 f. Dissertação (Mestrado em Ortodontia)-Universidade Federal do Rio de Janeiro, Rio de Janeiro, 1981

34. RICKETTS, R. M.; ROTH, R. H.; CHACONAS, S. J.; SCHULHOF, R. J.; ENGEL, G. A. Orthodontic diagnosis and planning [USA]: Rock Mountain Data Systems, 1982
35. RIEDEL, R. A. A review of retention problem. Angle Orthod Appleton, v. 30, no. 4, p. 179-193, Oct. 1960

36. RIEDEL, R. Retention and relapse. J Clin Orthod, Boulder, v. 10, p. 454-472, 1976

37. ROSSOUW, P. E.; PRESTON, C. B. A longitudinal evaluation of the anterior border of the dentition. Am J Orthod Dentofacial Orthop, St. Louis, v. 104, no. 2, p. 146-152, Aug. 1993.

38. SADOWSKY, C.; SCHNEIDER, B. J.; BEGOLE, E. A.; TAHIR, E. J. Long-term stability after orthodontic treatment: nonextraction with prolonged retention. Am J Orthod Dentofacial Orthop, St. Louis, v. 106, no. 3, p. 243-249, Sept. 1994

39. SHAPIRO, H. H. Growth in the mandibular dental arch. J Dent Res, Washington, D. C., v. 20, p. 335-340, 1941.

40. SHAPIRO, P. S. Mandibular dental arch form and dimension: treatment and post retention changes. Am J Orthod, St. Louis, v. 66, no. 1, p. 58-70, July 1974.

41. SHIELDS, T. E.; LITTLE, R. M.; CHAPKO, M. K. Stability and relapse of mandibular anterior alignment: a cephalometric appraisal of first premolar extraction cases treated by traditional Edgewise Orthodontics. Am J Orthod, St. Louis, v. 87, no. 1, p. 27-38, Jan. 1985

42. SINCLAIR, P. M.; LITTLE, R. M. Maturation of untreated norma occlusions. Am J Orthod, St. Louis, v. 83, no. 2, p. 114-123, Feb. 1983

43. SONDHI, A.; CLEALL, J. F.; BeGOLE, E. A. Dimensional changes in the dental archs of orthodontically treated cases. Am J Orthod, St. Louis, v. 77, no. 1, p. 60-74, Jan. 1980.

44. SOUTHARD, T. E.; BEHRENTS, R. G.; TOLLEY, E. A. The anterior component of occlusal force. Part 1. Measurement and distribution. Am J Orthod Dentofacial Orthop, St. Louis, v. 96, no. 6 p. 493-500, Dec. 1989

45. SOUTHARD, T. E.; BEHRENTS, R. G.; TOLLEY, E. A. The anterior component of occlusal force. Part 2: Relationship with dental malalignment. Am J Orthod Dentofacial Orthop, St. Louis, v. 97, no. 1, p. 41-44, Jan. 1990.

46. STEADMAN, S. R. Changes of intermolar and intercuspid distances following orthodontic treatment. Angle Orthod, Appleton, v. 31, no. 4, p. 207-215, Oct. 1961

47. STRANG, R. The fallacy of denture expansion as a treatment procedure. Angle Orthod, Appleton, v. 19, p. 12-22, 1949

48. STRANG, R. Factors associated with successful orthodontic treatment. Am J Orthod, St. Louis, v. 38, p. 790-800, 1952

49. UHDE, M.; SADOWSKY, C.; BEGOLE, E. A. Long-term stability of dental relationships after orthodontic treatment. Angle Orthod, Appleton, v. 53, no. 3, p. 240-252, July 1983.

50. VADEN, J. L.; GARDNER, R. L. Relapse revisted. Am J Orthod Dentofacial Orthop, St. Louis, v. 111, no. 5, p. 543-553, May 1997

51. WALTER, D. C. Comparative changes in mandibular canine and first molar widths. Angle Orthod, Appleton, v. 32, no. 4, p. $232-$ 240, Oct. 1962.

52. WERNER, S. P.; SHIVAPUJA, P. K.; HARRIS, E. F. Skeletodental changes in the adolescent accruing from use of the lip bumper. Angle Orthod, Appleton, v. 64, no. 1, p. 13-20, 1994. Discussion 21-22.

53. WILLIAMS, S.; ANDERSEN, C. E. Incisor stability in patients with anterior rotational mandibular growth. Angle Orthod, Appleton, no. 6, p. 431-442, 1995

Endereço de correspondência

Patricia Regina Rizzo Araújo

R. Leonor Calmon, 256, sala 301 - Cidade Jardim

CEP: 40.280-633 - Salvador-BA

E-mail: ptrizzo@bol.com.br 